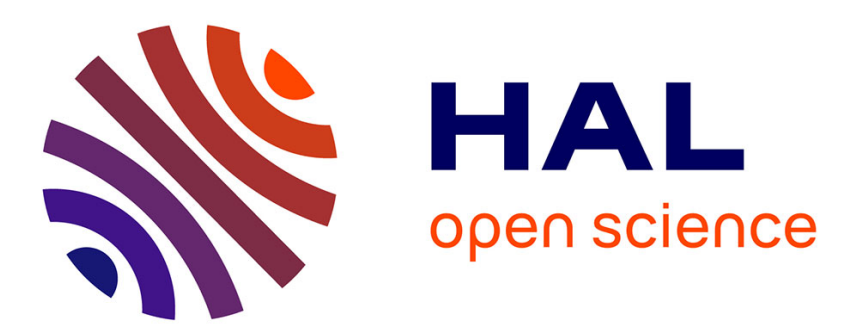

\title{
Transformation de phase dans l'aluminium induite par implantation ionique de nickel à haute énergie
}

\author{
R. Schäublin, R. Gotthardt
}

\section{To cite this version:}

R. Schäublin, R. Gotthardt. Transformation de phase dans l'aluminium induite par implantation ionique de nickel à haute énergie. Journal de Physique IV Proceedings, 1994, 04 (C3), pp.C3-297-C3303. 10.1051/jp4:1994341. jpa-00252538

\section{HAL Id: jpa-00252538 https://hal.science/jpa-00252538}

Submitted on 1 Jan 1994

HAL is a multi-disciplinary open access archive for the deposit and dissemination of scientific research documents, whether they are published or not. The documents may come from teaching and research institutions in France or abroad, or from public or private research centers.
L'archive ouverte pluridisciplinaire HAL, est destinée au dépôt et à la diffusion de documents scientifiques de niveau recherche, publiés ou non, émanant des établissements d'enseignement et de recherche français ou étrangers, des laboratoires publics ou privés. 


\title{
Transformation de phase dans l'aluminium induite par implantation ionique de nickel à haute énergie
}

\author{
R. SCHÄUBLIN et R. GOTTHARDT
}

Instituit de Génie Atomique, Ecole Polytechnique Fédérale de Lausanne, 1015 Lausanne, Switzerland

\begin{abstract}
Résumé: Implantation of $\mathrm{Ni}$ into a pure $\mathrm{Al}$ target leads to the formation of amorphous zones. Their formation is studied by transmission electron microscopy (TEM). The present results were obtained in samples implanted with ions having energies between 22 and $40 \mathrm{MeV}$ and fluences between $1.68 \times 10^{15}$ and $2.5 \times 10^{17} \mathrm{ions} / \mathrm{cm}^{2}$. In order to observe the profile of the ion implantation in the TEM, cross-sectional samples were thinned with a specially modified ion bombardement. Measurements of the atomic concentration have been carried out with energy dispersive spectroscopy (EDS). In spite of the Ni concentration (up to 3 at. \%) in the implanted layer, there is no observable nuclei of the $\mathrm{Al}_{3} \mathrm{Ni}$ phase after implantation at $120 \mathrm{~K}$ and room temperature. In contrary, amorphous zones with a diameter of about $10 \mathrm{~nm}$ are present together with a high density of dislocation loops and dislocations. The $\mathrm{Ni}$ concentration in the amorphous zones is about 25 at. \%. An implantation conducted at $520 \mathrm{~K}$ shows only crystalline precipitates of $\mathrm{Al}_{3} \mathrm{Ni}$. We conclude that the $\mathrm{Ni}$ implanted in $\mathrm{Al}$ agglomerates in zones having composition identical to that of the $\mathrm{Al}_{3} \mathrm{Ni}$ phase.
\end{abstract}

\section{Introduction}

L'implantation est un processus de modification des propriétés physiques et chimiques du matériau par l'introduction d'éléments appropriés sous la forme d'un faisceau de particules ionisées. Cette modification peut avoir lieu par la formation de nouvelles phases, la mise en ordre ou le désordre d'une phase ordonnée, la modification du diagramme de phase vers des solutions solides étendues ou l'amorphisation. Ce dernier aspect est l'objet de la présente étude. En général, les métaux purs et les solutions solides sont difficiles à amorphiser [1]. Toutefois l'amorphisation de solutions solides à été observée dans le cas d'implantations ioniques. A cause de son importance technologique, le système Al-Ni a été l'objet de nombreuses études [2,3]. L'étude de l'implantation du système $\mathrm{Al}-\mathrm{Ni}$ bénéficie donc d'une large quantité de données pour 
l'interprétation des résultats. L'emploi d'énergies relativement élevées permet de s'affranchir des effets de surfaces.

Différentes explications ont été proposées dans la littérature mais pour autant que l'on sache aucune n'est fondée sur des résultats expérimentaux rapportant à l'échelle nanométrique les débuts du processus. Ce travail présente les résultats d'une étude en microscopie électronique à transmission (MET) des débuts de la transformation cristal-amorphe dans I'Al par implantation de $\mathrm{Ni}$, c'est-à-dire pour une fraction amorphisée faible. L'observation en MET et en coupe transverse de la couche implantée a montré la présence de contrastes qui ont deux origines [4]. La première est la présence de défauts cristallins (boucles de dislocations et dislocations) et la deuxième est la présence de zones amorphes approximativement sphériques d'environ $10 \mathrm{~nm}$ de diamètre. Afin de déterminer les paramètres importants pour l'amorphisation, les observations ont été poursuivies sur des échantillons implantés à différentes doses et à différentes températures.

\section{Procédure expérimentale}

Les implantations ont été effectuées sur un accélérateur TANDEM situé à l'école polytechnique fédérale de Zürich (ETHZ). Les détails concernant la préparation des échantillons d'Al $5 \mathrm{~N}$ polycristallins pour l'implantation et la MET ayant été précisés dans une publication antérieure [4], seules les conditions d'implantations sont données ici. La première partie de ce travail concerne l'influence de la dose et de la température d'implantation sur l'amorphisation. Le tableau 1 donne les doses de $\mathrm{Ni}$ implanté, les énergies et les températures. La partie supérieure et inférieure du tableau donne les conditions pour l'étude de l'influence de la dose à température ambiante (TA) et de la température d'implantation à dose constante, respectivement. La dose donnée en pourcent atomique a été calculée au pic du profil d'implantation simulé par le programme TRIM [5]. La largeur du pic considérée - sur laquelle la concentration atomique est à peu près constante - est de $200 \mathrm{~nm}$. La profondeur et la largeur du pic d'implantation à mi-hauteur ont été mesurés sur les profils simulés par TRIM.

Pour étudier l'influence des cascades de déplacements induites par les ions sur les zones amorphes, un échantillon implanté à TA à la dose de $2.2 \%$ at. a été aminci pour la MET et post-implantés à TA à des doses croissantes. Les doses, en $\%$ at., de post-implantation données dans le tableau 2 correspondent respectivement à 2,20 et 300 fois la densité numérique de zones amorphes exprimée en concentration atomique. De plus, la concentration en Ni n'est pas fortement modifiée; l'augmentation est de $5 \%$ au maximum. L'énergie est relativement faible $(1.16 \mathrm{MeV})$ pour éviter la perte des ions traversants l'épaisseur (inférieure à $200 \mathrm{~nm}$ ) des zones transparentes aux électrons. De surcroît, un film d'Al de $740 \mathrm{~nm}$ d'épaisseur à été placé avant l'échantillon pour ralentir les ions. Une observation détaillée des micrographies prises dans une même région à des doses différentes a été faite pour suivre l'évolution des zones amorphes. 
Tableau 1: Conditions d'implantation à haute énergie du $\mathrm{Ni}$ dans I'Al (TA : température ambiante).

\begin{tabular}{c|c|c|c|c|} 
Dose: [ions $\left./ \mathrm{cm}^{2}\right]$ & {$[\%$ at] } & Prof.-larg. $[\mu \mathrm{m}]$ & Energie [MeV] & Température [K] \\
\hline $2.50 \times 10^{17}$ & ${ }^{*} 6.1 \pm 0.5$ & $7.14-0.55$ & 27.7 & TA \\
\hline $8.38 \times 10^{16}$ & $2.2 \pm 0.1$ & $7.12-0.55$ & 27.5 & TA \\
\hline $2.57 \times 10^{16}$ & $0.68 \pm 0.03$ & $7.12-0.55$ & 27.5 & TA \\
\hline \hline $2.50 \times 10^{16}$ & $0.59 \pm 0.03$ & $6.26-0.50$ & 22 & 120 \\
\hline $2.50 \times 10^{16}$ & $0.59 \pm 0.03$ & $6.26-0.50$ & 22 & 530 \\
\hline
\end{tabular}

"Elargissement du pic du à une contamination accidentelle de surface; dose réelle: $3.05 \pm 0.50 \%$ at.

Tableau 2: Conditions d'implantation à TA des échantillons amincis pour la MET.

\begin{tabular}{c|c|} 
Dose: $\left[\right.$ ions $\left./ \mathrm{cm}^{2}\right]$ & {$[\% \mathrm{at}]$} \\
\hline $1.6 \times 10^{13}$ & $7.0 \times 10^{-4} \pm 0.7 \times 10^{-4}$ \\
\hline $1.6 \times 10^{14}$ & $7.0 \times 10^{-3} \pm 0.7 \times 10^{-3}$ \\
\hline $2.5 \times 10^{15}$ & $0.10 \pm 0.01$ \\
\hline
\end{tabular}

Afin de mesurer la concentration atomique de $\mathrm{Ni}$ dans les zones amorphes par rapport à la concentration atomique de $\mathrm{Ni}$ de la matrice $\mathrm{Al}$ pour l'échantillon implanté à la dose maximale, la méthode EDS (Energy Dispersive Spectroscopy) a été utilisée. La mesure EDS est effectué dans un MET et consiste à analyser les rayons $X$ émis lors de la désexcitation des atomes de l'échantillon qui ont été excités par les électrons. Cette analyse permet de déterminer la nature des éléments présents dans l'échantillons ainsi que la quantité relative d'un élément donné. Les zones amorphes observées ont un diamètre maximal d'environ $10 \mathrm{~nm}$ à la dose maximale. II est donc nécessaire de faire la mesure avec un faisceau d'électrons dont le diamètre est inférieur à $10 \mathrm{~nm}$. La mesure a été effectuée à l'aide d'un MET HITACHI HF 2000 à canon à émission de champ car il permet de travailler avec une sonde de $5 \mathrm{~nm}$ de diamètre.

\section{Résultats}

La figure 1 présente les micrographies en MET de l'évolution de la phase amorphe en fonction de la dose. $\|$ apparaît que les zones amorphes sont approximativement sphériques, et que leur taille augmente lorsque la dose augmente.

La fraction volumique rendue amorphe $(\alpha)$ par l'implantation est calculée à partir de la mesure sur les micrographies de la densité numérique par unité de surface et du diamètre moyen des zones amorphes. Les zones amorphes sont supposées sphériques. La densité numérique par unité de volume de zones amorphes est égale à la densité par unité de surface divisée par l'épaisseur de la région de mesure. L'épaisseur est mesurée par stéréoscopie. La fraction amorphisée $(\alpha)$ est alors donnée par le produit de la densité numérique et du volume moyen des zones amorphes. 
Le tableau 3 présente les résultats des mesures en fonction de la dose. II apparaît que la densité ne manifeste pas une dépendance monotone avec la dose. La taille des zones amorphes ainsi que la fraction amorphe augmentent lorsque la dose augmente.

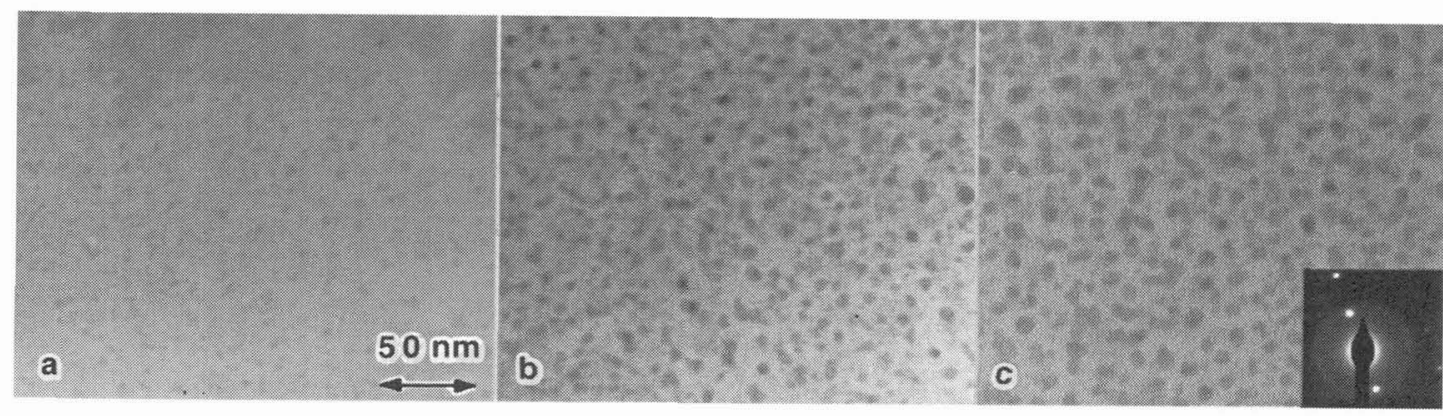

Figure 1: Micrographies en MET de la couche implantée dans I'Al à TA montrant les zones amorphes (disques sombres) à une dose d'implantation de a) 0.68 b) 2.2 et c) $3.05 \%$ at. de Ni. Epaisseurs respectives des lames minces: 38, 36 et $26 \mathrm{~nm}$.

Tableau 3: Densité numérique, diamètre moyen des zones amorphes et fraction amorphe $\alpha$ en fonction de la dose.

\begin{tabular}{|c|c|c|c|} 
Dose [\% at.] & Densité $\left[\mathrm{m}^{-3}\right]$ & $\emptyset$ moy. [nm] & $\alpha$ \\
\hline 0.68 & $1.17 \times 10^{23}$ & 4.9 & 0.007 \\
\hline 2.2 & $2.1 \times 10^{23}$ & 6.64 & 0.032 \\
\hline 3.05 & $1.47 \times 10^{23}$ & 8.14 & 0.042 \\
\hline
\end{tabular}

La figure 2 présente des micrographies de MET d'échantillons implantés à une dose de $0.6 \%$ at. et à différentes températures. L'amorphisation a lieu à $120 \mathrm{~K}$ et à TA. A basse température cependant, il apparaît que la taille et la densité numérique des zones amorphes est plus faible qu'à TA.

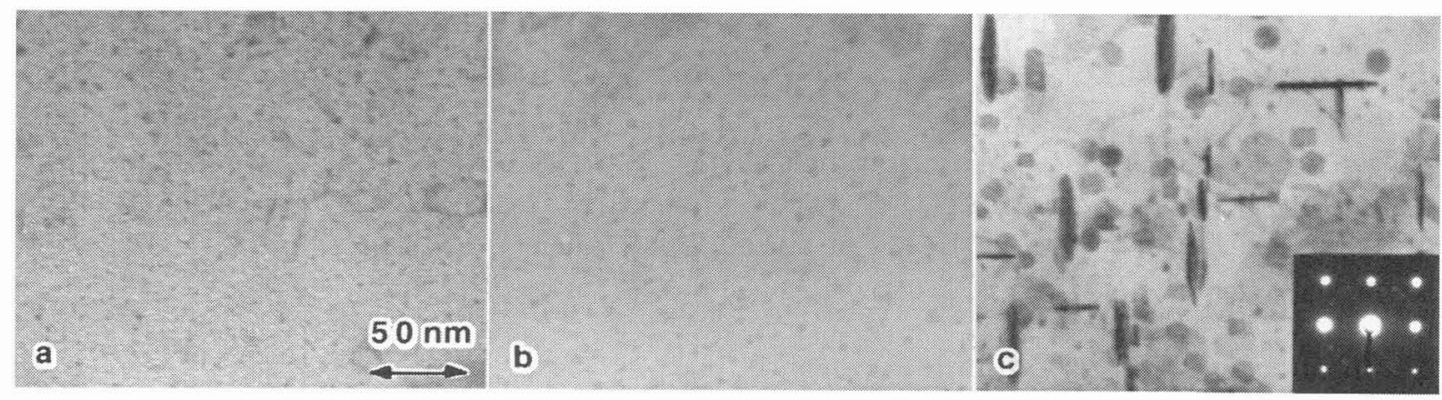

Figure 2: Micrographies en MET d'échantillons d'Al implantés à environ $0.6 \%$ at. de $\mathrm{Ni}$ et à une température de a) $120 \mathrm{~K}$, b) TA et c) $520 \mathrm{~K}$. Epaisseurs respectives: 43,38 et $79 \mathrm{~nm}$.

A $520 \mathrm{~K}$ l'amorphisation n'a pas lieu, mais par contre, il apparaît des précipités cristallins en forme soit de disque d'en moyenne $50 \mathrm{~nm}$ de diamètre et d'environ $4 \mathrm{~nm}$ d'épaisseur et parallèles aux plans cubiques de l'Al, soit de sphère ou de 
cube d'une taille d'environ $13 \mathrm{~nm}$. Le tableau 4 montre les valeurs de la densité, de la taille des zones amorphes et de la fraction amorphe en fonction de la température d'implantation. La fraction amorphe à basse température est d'un ordre de grandeur inférieur à la fraction amorphe à TA.

Tableau 4: Densité, taille des zones amorphes et fraction amorphe en fonction de la température d'implantation pour une dose de $0.6 \%$ at.

\begin{tabular}{l|c|c|} 
& $120 \mathrm{~K}$ & TA \\
\hline Densité $\left[\mathrm{za} / \mathrm{m}^{3}\right]$ & $1.4 \times 10^{23}$ & $2.1 \times 10^{23}$ \\
\hline$\emptyset$ moy. $[\mathrm{nm}]$ & 3.0 & 6.6 \\
\hline$\alpha$ & 0.002 & 0.032 \\
\hline
\end{tabular}

La figure 3 présente des micrographies de zones amorphes présentes dans l'échantillon implanté à $2.2 \%$ at. et post-implanté aux 2 doses les plus élevées. Certaines petites zones amorphes disparaissent (voir cercle p. ex.). Toutefois la majorité des zones amorphes (voir flèches p. ex.) ne disparaît pas et ne change pas significativement de taille, alors qu'en moyenne il arrive 300 cascades par zone pour la dose la plus élevée. Une cascade seule ne peut donc pas recristalliser une zone amorphe, car sinon la configuration des zones amorphes sur les micrographies serait drastiquement modifiée après post-implantation.

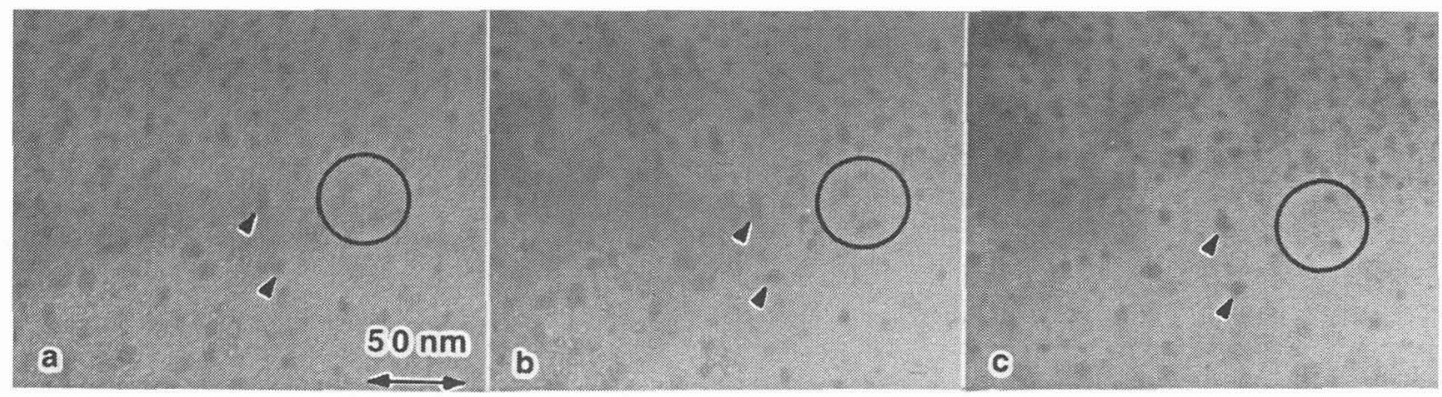

figure 3: Micrographies en MET d'un échantillon d'Al implanté à TA à une dose de a) $2.2 \%$ at. et post-implanté à une dose de b) 0.007 et c) $0.1 \%$ at. de Ni.

Les mesures EDS ont été faites sur des zones amorphes et entre les zones amorphes de l'échantillon implanté à $3.05 \%$ at. La zone amorphe considérée a un diamètre de 10 à $12 \mathrm{~nm}$ et l'épaisseur de la région la contenant est de 30 à $20 \mathrm{~nm}$ respectivement. Il apparaît que le taux de comptage du signal Ni est 5 fois plus élevé lors de la mesure sur les zones amorphes que lors de la mesure dans la matrice. Un nombre de total de 12 mesures, dont 8 sur les zones amorphes, a été effectué. Les concentrations atomiques de $\mathrm{Ni}$ dans les zones amorphes et dans la matrice sont en moyenne de 25 et $2.1 \%$ at., respectivement. La composition des zones amorphes est donc celle d'un composé $\mathrm{Al}_{75} \mathrm{Ni}_{25}$. 


\section{Discussion}

Deux processus ont été proposés pour décrire l'amorphisation des systèmes amorphisables. Le premier invoque la cascade de déplacements d'atomes induite par le ion incident. Cette cascade donne lieu à un pic thermique. La région est alors considérée comme une gouttelette liquide [6]. Le refroidissement rapide de la zone en fusion pourrait alors induire l'amorphisation [7]. Le deuxième mécanisme est l'accumulation de défauts ponctuels introduits par l'implantation. Ces défauts ponctuels peuvent augmenter l'énergie élastique du cristal suffisamment pour rendre la phase amorphe favorable [8].

Nos résultats indiquent que la fraction amorphe augmente avec la dose. Cela est dû d'une part à l'augmentation du nombre de déplacements par atome et d'autre part à l'augmentation du nombre de $\mathrm{Ni}$ introduits qui stabilisent la phase amorphe [3]. Ce dernier effet semble prédominant sur le précédent au vu des résultats à basse température et de post-implantation. A basse température la densité et la taille des zones amorphes sont plus faibles qu'à TA pour une dose équivalente. La diffusion de $\mathrm{Ni}$ étant réduite à basse température, le nombre et la taille de zones riches en $\mathrm{Ni}$ sont diminués et le nombre de zones amorphes aussi. De plus les résultats de l'expérience de post-implantation nous indiquent qu'une cascade seule ne peut modifier une zone amorphe. Ainsi la formation des germes d'amorphisation dépend essentiellement de la concentration locale de $\mathrm{Ni}$.

Les résultats de la mesure EDS indiquent que le contenu des zones amorphes a la composition de la phase $\mathrm{Al}_{3} \mathrm{Ni}$. II y a donc une force motrice qui tend à diminuer l'énergie du cristal cible en formant des zones localement riche en $\mathrm{Ni}$. Le diagramme de phase du système $\mathrm{Ni}$-Al indique que thermodynamiquement, dans le cas d'une solution solide d'Al, il tend à former une phase $\mathrm{Al}_{3} \mathrm{Ni}$. Les observations en MET montrent que la phase cristalline n'est pas formée à $120 \mathrm{~K}$ et à TA. La cible étant constamment irradiée pendant le processus d'agglomération du $\mathrm{Ni}$, les déplacements atomiques induits empêchent la cristallisation de $\mathrm{Al}_{3} \mathrm{Ni}$. Cependant, à $520 \mathrm{~K}$ on observe la phase cristalline. A cette température, la diffusion est suffisante pour établir un ordre à courte distance durant I'rradiation, c'est-à-dire que la mise en ordre par diffusion thermique est plus rapide que la mise en désordre balistique.

Pour comprendre l'amorphisation, par implantation, de zones dont la composition est $\mathrm{Al}_{3} \mathrm{Ni}$ quelques résultats de la littérature sont reportés ici. Les systèmes ion-cible qui ont tendance à former des composés stoechiométriques cristallins sont plus à même d'être rendus amorphes [9]. Ainsi le système immiscible $\mathrm{Pb}-\mathrm{Ni}$, qui ne présente pas de composé cristallin, n'est pas amorphisable, alors que les systèmes $\mathrm{B}-\mathrm{Ni}$, Ni-Al, Fe-Al, qui ont des composés cristallins, le sont [9]. Dans le cas du système $\mathrm{Ni}-\mathrm{Al}$, il apparaît que la phase $\mathrm{Al}_{3} \mathrm{Ni}$ est facilement amorphisable autant avec des ions qu'avec des électrons [10]. De plus, il a été montré que l'amorphisation de $\mathrm{Al}_{3} \mathrm{Ni}$ par les ions pourrait être induite directement par les cascades [10]. Cela signifie que l'Al $3 \mathrm{Ni}$ désordonné a une énergie libre supérieure à un amorphe $\mathrm{Al}_{75} \mathrm{Ni}_{25}$. 


\section{Conclusion}

Les implantations à haute énergie permettent d'atteindre des profondeurs inhabituelles. A l'aide de techniques de préparation appropriées [4], les échantillons sont préparés en coupe transverse pour la MET. Les observations conduites en MET permettent de caractériser les germes d'amorphes présents à des doses relativement faibles d'implantation.

L'implantation à température ambiante de $\mathrm{Ni}$ dans l'Al à haute énergie (27.5 à $27.7 \mathrm{MeV}$ ) pour des doses comprises entre 0.68 et $3.05 \%$ at. conduit à la formation d'une phase amorphe sous la forme de zones approximativement sphériques d'un diamètre maximal d'environ $10 \mathrm{~nm}$. La mesure de concentration atomique de $\mathrm{Ni}$, effectuée sur l'échanitllon implanté à une dose de $3.05 \%$ at., monte que les zones amorphes contiennent un mélange $\mathrm{Ni}$-Al dont la composition est $\mathrm{Al}_{75} \mathrm{Ni}_{25}$. La matrice est appauvrie et contient $2.1 \%$ at. de Ni. L'implantation à $120 \mathrm{~K}$ montre une fraction amorphe diminuée par rapport à l'implantation à température ambiante, et une implantation à $520 \mathrm{~K}$ crée la phase cristalline $\mathrm{Al}_{3} \mathrm{Ni}$.

II semble donc que le $\mathrm{Ni}$ implanté dans l'Al n'est pas distribué homogènement mais est concentré dans des zones dont la composition est celle de la phase $\mathrm{Al}_{3} \mathrm{Ni}$. Ces zones sont amorphes à 120 et $300 \mathrm{~K}$, et cristallines à $520 \mathrm{~K}$.

\section{Remerciements}

Nous sommes très reconnaissants aux docteurs $M$. Suter et M. Döbeli de I'ETH de Zürich pour la direction des implantations.

\section{Références}

[1] E. M. Schulson, J. of Nuclear Mater. 83 (1979) 239-264.

[2] C. Jaouen, J.P. Rivière and J. Delafond, Nuclear Instrum. and Methods in Phys. Res. B 19/20 (1987) 549-553.

[3] A. Benyagoub, L. Thomé, Phys. Rev. B 3815 (1988) 10205-10216.

[4] R.Schäublin and R. Gotthardt, Radiation Effects and Defects in Solids 126 (1993) 185-188.

[5] Ziegler J. F., Biersack J. P. and Littmark U., "The Stopping and Ranges of lons in Matter", Pergamon press, (1985).

[6] M. Alurralde, A. Caro, M. Victoria, J. of Nucl. Mater. 183 1-2 (1991) 33-39.

[7] W. L. Johnson, in "Materials Modification by High-fluence lon Beams", R. Kelly and M. Fernanda (eds.) (1989) 405-419.

[8] G. Martin and A. Barbu, in "Decomposition of Alloys: The Early Stages", Pergamon Press (1984) 70-76.

[9] A. Ali, W. A. Grant, P.J. Grudy, Phil. Mag. B 373 (1978) 353-376.

[10] M. Nastasi, J.M. Williams, E.A. Kenik, J.W. Mayer, Nuclear Instrum. and Methods in Phys. Res. B 19/20 (1987) 543-548. 